\title{
Prognostic value of heart rate turbulence for risk assessment in patients with unstable angina and non-ST elevation myocardial infarction
}

This article was published in the following Dove Press journal:

Vascular Health and Risk Management

9 August 2013

Number of times this article has been viewed

\section{Patricia RE Harris' \\ Phyllis K Stein ${ }^{2}$ \\ Gordon L Fung ${ }^{3}$ \\ Barbara J Drew ${ }^{4}$}

'Electrocardiographic Monitoring Research Laboratory, School of Nursing, Department of Physiological Nursing, University of California, San Francisco, CA, USA; ${ }^{2}$ Heart Rate Variability Laboratory, School of Medicine, Division of Cardiology, Washington University, St Louis, MO, USA; ${ }^{3}$ Cardiology Services, Department of Medicine, ${ }^{4} \mathrm{School}$ of Nursing, Department of Physiological Nursing, Division of Cardiology, University of California, San Francisco, CA, USA
Correspondence: Patricia RE Harris School of Nursing, University of California, 2 Koret Way, San Francisco, CA 94143-0610, USA

Tel +l 4154766089

Email patriciarae.harris@ucsf.edu
Background: We sought to examine the prognostic value of heart rate turbulence derived from electrocardiographic recordings initiated in the emergency department for patients with non-ST elevation myocardial infarction (NSTEMI) or unstable angina.

Methods: Twenty-four-hour Holter recordings were started in patients with cardiac symptoms approximately 45 minutes after arrival in the emergency department. Patients subsequently diagnosed with NSTEMI or unstable angina who had recordings with $\geq 18$ hours of sinus rhythm and sufficient data to compute Thrombolysis In Myocardial Infarction (TIMI) risk scores were chosen for analysis $(n=166)$. Endpoints were emergent re-entry to the cardiac emergency department and/or death at 30 days and one year.

Results: In Cox regression models, heart rate turbulence and TIMI risk scores together were significant predictors of 30-day (model chi square 13.200, $P=0.001$, C-statistic 0.725 ) and one-year (model chi square 31.160, $P<0.001$, C-statistic 0.695) endpoints, outperforming either measure alone.

Conclusion: Measurement of heart rate turbulence, initiated upon arrival at the emergency department, may provide additional incremental value in the risk assessment for patients with NSTEMI or unstable angina.

Keywords: acute coronary syndrome, electrocardiographic monitoring, heart rate turbulence, non-ST elevation myocardial infarction, outcomes, prognosis, unstable angina

\section{Introduction}

Patients who present to a hospital emergency department with ischemic discomfort that is subsequently diagnosed as unstable angina or non-ST elevation myocardial infarction (NSTEMI) are a heterogeneous group in terms of risk for death or nonfatal cardiac events. The most recent guidelines for the management of patients with unstable angina or NSTEMI ${ }^{1}$ recommend the use of risk stratification tools such as the Thrombolysis In Myocardial Infarction (TIMI) risk score ${ }^{2}$ to assess the patient's likelihood of future adverse cardiac events.

Antman et al developed the TIMI risk score composed of seven (one-point) risk indicators rated on presentation to the emergency department. The composite endpoints (all-cause mortality, new or recurrent myocardial infarction, or severe recurrent ischemia prompting urgent revascularization within 14 days) increase as the TIMI risk score increases. Additional research has demonstrated the accuracy of the TIMI risk score in predicting 30-day ${ }^{3,4}$ and one-year ${ }^{5}$ outcomes, including myocardial infarction or cardiovascular death, in patients with cardiac symptoms seen in the emergency department who do not have ST elevation on the electrocardiogram. 
Because all patients who present to the emergency department with symptoms suggestive of acute coronary syndrome receive continuous electrocardiographic (ECG) monitoring as part of their routine care, we sought to determine if the ECG measure heart rate turbulence could be used to provide prognostic information. Heart rate turbulence refers to fluctuations in the cardiac cycle in response to a ventricular premature complex (VPC). ${ }^{6}$ The two phases of oscillation, acceleration followed by deceleration, are measured using parameters termed turbulence onset and turbulence slope. The two heart rate turbulence parameters examine the period encompassing at least two sinus RR intervals prior to a ventricular beat, the VPC itself, the ensuing compensatory pause, and the subsequent 15 sinus intervals. To calculate turbulence onset and/or turbulence slope, at least five VPCs are required during a 24-hour ECG recording. ${ }^{\text {? }}$

Abnormal heart rate turbulence in healed myocardial infarction, measured 2-3 weeks after the index event, has been shown to identify individuals who are 3.2 times more likely to die during 21 months of follow-up. ${ }^{8}$ Heart rate turbulence has also been examined in recordings started immediately after admission of patients with myocardial infarction to a coronary care unit, and abnormal measures have been associated with higher risk of mortality up to 360 days. ${ }^{9}$ In patients with unstable angina, abnormal heart rate turbulence measured within 24 hours of admission has been shown to confer an increased risk for cardiovascular mortality over the ensuing six months. ${ }^{10}$ What remains unknown is whether abnormal heart rate turbulence measured at the earliest possible phase of care for acute coronary syndrome when a patient is initially attached to a cardiac monitor in the emergency department would have prognostic value. If heart rate turbulence has prognostic value during the earliest phase of care, future cardiac monitor algorithms could be designed to measure this parameter and augment the traditional TIMI risk tools, potentially providing a basis for therapeutic decision-making.

The opportunity to investigate heart rate turbulence during the earliest phase of care was available to us from our Ischemia Monitoring and Mapping in the Emergency Department In Appropriate Triage and Evaluation of Acute Ischemic Myocardium (IMMEDIATE AIM) study, a prospective clinical trial of 1308 patients presenting to our institution's emergency department with symptoms of acute coronary syndrome and funded by the National Institutes of Health (RO1HL69753). Twenty-four-hour 12-lead ECG recordings were started in the emergency department for 1308 patients with a median door to ECG start time of 45 minutes (median time from symptom onset was four hours). While all patients with symptoms of acute coronary syndrome need immediate evaluation, unstable angina and NSTEMI are related conditions, and clinical management for these patients is distinctly different from that in those with clear ischemic ST-elevation changes. ${ }^{1}$ Thus, the aim of our analysis was to determine whether abnormal heart rate turbulence, measured in the initial 24 hours after presentation to the emergency department, in patients with symptoms of acute coronary syndrome but no ST elevation, would provide prognostic information equal to or above and beyond the TIMI risk score for shortterm (30-day) or long-term (one-year) outcomes, identified as the composite endpoint of cardiac-related readmission to the emergency department, cardiac rehospitalization, and/ or cardiac death.

\section{Materials and methods Sample and data collection}

Of 1308 patient visits to the emergency department for symptoms of acute coronary syndrome, there were 1153 unique patients enrolled from 2002 to 2004. Verbal assent was obtained at the time of recruitment, and written consent was obtained from the patient or surrogate after stabilization. We placed 24-hour Holter recorders (H-Scribe System, Mortara Instrument, Milwaukee, WI, USA) as soon as possible after the patients arrived in the emergency department. Trained research nurses applied the ECG leads, supervised monitoring, and downloaded data to the H-Scribe review station. Radiolucent electrodes and lead wires were used to aid uninterrupted monitoring during procedures such as cardiac catheterization. The University of California, San Francisco institutional review board approved the study. The study design and data collection have been detailed elsewhere. ${ }^{11}$ Patients' demographic and clinical information was gathered upon enrollment or extracted later via chart review.

\section{Follow-up}

We followed patients for one year after their initial visit to the emergency department and hospitalization. Data regarding patients' emergency department or hospital readmissions and survival were collected via telephone calls, medical records, and the public access social security mortality database. Information on one-year survival and rehospitalization was obtained for all patients in the current analysis.

\section{Inclusion and exclusion criteria}

ECG recordings with at least 18 hours of normal sinus beats, which represented individuals admitted to hospital to rule out 
myocardial infarction and who received a discharge diagnosis of unstable angina or NSTEMI were included in the analysis. NSTEMI was identified by ST-segment depression or T-wave inversion, a positive serum troponin biomarker, absence of ST-segment elevation, and chest pain or equivalent angina symptoms. ${ }^{1}$ Unstable angina was defined as "evidence of coronary occlusion without myocardial infarction," in accordance with the Diagnosis Related Group criteria for "angina pectoris, intermediate coronary syndrome." Recordings were automatically scanned, manually edited, and annotated. Patients with sufficient clinical data to compute a TIMI score were included in the final analysis.

Patients with atrial fibrillation or pacing devices were excluded. Patients for whom we did not have at least one year of follow-up information were also excluded, as were those whose emergency department records lacked enough clinical data to compute a TIMI risk score.

\section{Endpoints}

Recent research has identified the heavy burden of hospital readmission for patients diagnosed with acute coronary syndrome, including those with unstable angina and NSTEMI. ${ }^{12}$ Therefore, the primary endpoint comprised a composite of cardiac-related events within 30 days and one year of discharge from the emergency department, including return to the emergency department with cardiac symptoms and subsequent cardiac diagnosis, cardiac rehospitalization, and/or cardiac death. Treatment in the emergency room with a cardiac diagnosis (whether followed by transfer to hospital cardiac care or not) was considered an emergent re-entry. Prescheduled cardiac procedures were not included in the analysis.

Patients with more than one event (specifically, patients who were readmitted and later died) were included in the analysis one time only. Return to the emergency department or rehospitalization was considered cardiac if the patient was diagnosed with a recurring episode of acute coronary syndrome and/or heart failure. Death was considered to be due to a cardiac cause if sudden cardiac death, acute myocardial infarction, or heart failure was reported in the hospital record or by family members or patients in the follow-up phone calls. Whenever possible, patients' or family members' statements were confirmed by data in the medical record. We also looked at each endpoint individually, ie, readmission or death due to a cardiac cause.

\section{Demographic and clinical variables}

Demographic information was collected, including gender, race, comorbidities. Clinical information included comorbidities and history of coronary artery disease.
The seven parameters required to calculate a TIMI risk score were obtained from the patient's emergency department record, cardiac catheterization report, and/or discharge summary, and included: age ( $\geq 65$ years); number of risk factors for coronary artery disease $(\geq 3)$; known coronary artery stenosis $>50 \%$; use of aspirin within the previous seven days; angina episodes within the previous 24 hours; ST changes $>0.5 \mathrm{~mm}$; and positive cardiac markers. ${ }^{2}$

Each risk parameter was assigned one point, whereby risk increases with ascending values. For this analysis, TIMI risk scores were divided into six categories; patients with 0 or 1 risk factor were grouped together into one low-risk category, and patients with scores of 6-7 were placed into the highest risk category, in accordance with the literature. ${ }^{2}$ Categories 2 through 5 remained the same. If extent of coronary disease was unknown, the default position was to count the parameter as " 0 ". If aspirin was listed as a routine medication, the default position was to count the parameter as " 1 " (unless there was mention in the patient's chart that the medication had not been taken); if there was no mention of aspirin in the chart, the parameter was counted as " 0 ". Beyond these default decisions, if data for computing the TIMI score were missing, the score was not computed and the participant was not included in the analysis.

\section{Electrocardiographic monitoring variables}

Variables derived from 24-hour Holter monitoring, including mean heart rate and number of VPCs, were calculated, in addition to heart rate turbulence. The ECG sampling rate was 180 samples per second. ECG recordings were automatically scanned and manually edited using $\mathrm{H}$-Scribe analysis software at the ECG Monitoring Research Laboratory, University of California, San Francisco School of Nursing. ECG research software, validated by the Heart Rate Variability Laboratory, Washington University School of Medicine, St Louis, MO, USA, was used to verify eligible recordings and compute heart rate turbulence.

\section{Analysis of heart rate turbulence}

To obtain variables for heart rate turbulence, RR intervals were exported as text files and transformed via a dedicated MatLab program (Mathworks, Natick, MA, USA) for compatibility with the research software. ECG recordings of patients in sinus rhythm with $\geq 25$ VPCs during the recording time were used in the analysis, in accordance with research by Bauer et al. ${ }^{8}$ Values for turbulence onset and turbulence slope were derived by averaging responses to the VPCs, which according to Bauer et al, must be based on no less 
than five VPCs to be considered reliable for assessment of heart rate turbulence. ${ }^{?}$

Turbulence onset, an indicator of early sinus acceleration after a VPC, was calculated by measuring the differences between two sinus beats following a compensatory pause $\left(R_{1}\right.$ and $\left.R R_{2}\right)$ and the two sinus beats immediately prior to the VPC $\left(R_{-1}\right.$ and $\left.R R_{-2}\right)$, using the formula: $\left\{\left[\left(\mathrm{RR}_{1}+\mathrm{RR}_{2}\right)-\left(\mathrm{RR}_{-1}+\mathrm{RR}_{-2}\right)\right] /\left(\mathrm{RR}_{-1}+\mathrm{RR}_{-2}\right) \times 100\right\}$, expressed as a percentage..$^{7-9}$

Turbulence slope, an indicator of late sinus deceleration after a VPC, was measured as the maximum positive slope of all slopes in a series of regression lines derived from five consecutive sinus beats within the first 15 sinus beats after the VPC, and expressed in msec per RR interval. ${ }^{7-9}$

Dichotomous heart rate turbulence variables were created using cutpoints for normal values according to published clinical standards, ie, turbulence onset $<0.0 \%$ and turbulence slope $>2.5 \mathrm{msec}$ per RR interval. Risk categories were established according to published measurement standards as follows:

- $\quad$ low risk $=$ "normal" heart rate turbulence variables (turbulence onset $<0.0 \%$ and turbulence slope $>2.5 \mathrm{msec}$ per RR interval)

- medium risk $=$ one "abnormal" heart rate turbulence variable (turbulence onset $\geq 0.0 \%$ or turbulence slope $\leq 2.5 \mathrm{msec}$ per RR interval)

- high risk $=$ two abnormal heart rate turbulence variables (turbulence onset $\geq 0.0 \%$ and turbulence slope $\leq 2.5 \mathrm{msec}$ per RR interval). ${ }^{7}$

\section{Statistical analysis}

Descriptive statistics were used to examine the demographic and clinical variables. To assess proportional hazard across time, univariate Cox regression survival analyses were performed for each outcome using TIMI or heart rate turbulence risk scores. Harrell's cumulative index (the C-statistic, a measure of concordance) was calculated to evaluate the predictive ability of the models. ${ }^{13}$

Heart rate turbulence and TIMI variables and risk scores were retested within multivariate regression models in relation to outcomes. Alpha was set at $<0.05$. C-statistics were generated again to reassess the predictive ability of the models.

Bootstrapping (1000 samples, 95\% confidence interval $[\mathrm{CI}]$ ) was used to examine model stability (alpha $<0.05){ }^{14}$ Analyses were performed using International Business Machines statistical software (Statistical Package for Social Sciences version 19, IBM, Armonk, NY, USA).

\section{Results}

\section{Patient characteristics}

Of 1153 unique patient recordings, we determined that 256 represented patients diagnosed with unstable angina; 173 recordings had $\geq 18$ hours of analyzable sinus rhythm. Seven patients were missing parameters beyond the default positions required to compute the TIMI score. Complete ECG, clinical, and follow-up data was available for 166 patients. For this subgroup of IMMEDIATE AIM patients, the median ECG Holter recording time was 1439 minutes; median time between symptom onset and Holter placement was four hours and 15 minutes; and median door-to-Holter time was 46 minutes. The median length of hospital stay was three (1-26) days; 41 patients (25\%) stayed in the hospital for one day. The median follow-up period for survivors was 368 days.

The majority of patients had a prior history of coronary artery disease $(n=115,69 \%)$. Among these patients, 76 had had a previous myocardial infarction (54\%); 64 had had a previous percutaneous coronary intervention (39\%), and 37 had had a coronary artery bypass graft (22\%). One hundred and eleven patients had had one or more cardiac catheterizations (67\%). Table 1 shows additional patient characteristics.

\section{Outcomes}

Within 30 days of discharge from the emergency department, $140(84 \%)$ patients had event-free outcomes and $26(16 \%)$ experienced adverse cardiac events. After the first 30-day period, an additional 59 patients (36\%) returned to the emergency department and received a cardiac diagnosis; 44 of these patients were readmitted to hospital. Four further patients died from a cardiac-related cause. Over the course of the follow-up year, 77 (46\%) patients remained event-free. Patient outcomes are shown in Table 2.

\section{TIMI and heart rate turbulence risk scores}

TIMI and heart rate turbulence risk scores were computed for each patient. The numbers of patients within each category are listed in Table 3 (TIMI) and Table 4 (heart rate turbulence). Twenty-seven patients had too few VPCs to calculate turbulence onset or turbulence slope. To examine the usefulness of the turbulence measures individually, turbulence onset dichotomized at $0 \%$ and turbulence slope dichotomized at $2.5 \mathrm{msec}$ per RR interval were tested in a univariate Cox regression model, and both were significant 
Table I Characteristics of patients with unstable angina or NSTEMI

\begin{tabular}{|c|c|c|c|c|}
\hline \multirow[t]{2}{*}{ Variable } & \multirow{2}{*}{$\begin{array}{l}\text { Patients } \\
(n=166)\end{array}$} & \multicolumn{2}{|c|}{ Patients with cardiac-related events (n) } & \multirow{2}{*}{$\begin{array}{l}\text { Event-free patients } \\
(n=77)\end{array}$} \\
\hline & & $\begin{array}{l}30 \text { days } \\
(n=26)\end{array}$ & $\begin{array}{l}\text { One year, including } \\
30 \text {-day outcomes } \\
(n=89)\end{array}$ & \\
\hline Mean age, years & $65 \pm 13$ & $65 \pm 12$ & $66 \pm 13$ & $65 \pm 12$ \\
\hline Male & $91(55 \%)$ & 13 & 44 & 47 \\
\hline \multicolumn{5}{|l|}{ Race } \\
\hline African American & $31(19 \%)$ & 4 & 24 & 7 \\
\hline Asian & 44 (27\%) & 9 & 20 & 24 \\
\hline Caucasian & $79(48 \%)$ & 12 & 36 & 43 \\
\hline American Native/Pacific Islander & $12(7 \%)$ & 1 & 9 & 3 \\
\hline Latino ethnicity, $n$ (\% of total) & $15(9 \%)$ & 2 & 12 & 3 \\
\hline \multicolumn{5}{|l|}{ ACS diagnoses } \\
\hline Unstable angina & $126(64.7 \%)$ & 21 & 72 & 54 \\
\hline NSTEMI & $40(24.6 \%)$ & 5 & 17 & 23 \\
\hline \multicolumn{5}{|l|}{ History or comorbidity } \\
\hline Prior history of coronary artery disease & $115(69 \%)$ & 19 & 70 & 45 \\
\hline Family history of coronary artery disease & $88(53 \%)$ & 12 & 41 & 47 \\
\hline Diabetes & $44(27 \%)$ & 12 & 30 & 14 \\
\hline Hypertension & $128(77 \%)$ & 18 & 73 & 55 \\
\hline Hypercholesterolemia & $113(68 \%)$ & 16 & 64 & 49 \\
\hline Current smoker & $30(18 \%)$ & 3 & 17 & 13 \\
\hline \multicolumn{5}{|l|}{ Inhospital therapy } \\
\hline Beta-blocker & l 47 (89\%) & 23 & 78 & 69 \\
\hline Percutaneous coronary intervention & $52(31 \%)$ & 10 & 27 & 25 \\
\hline Coronary artery bypass graft & $12(7 \%)$ & 3 & 6 & 6 \\
\hline \multicolumn{5}{|l|}{ 24-hour electrocardiographic data } \\
\hline Mean heart rate, beats per minute & $65 \pm 11$ & $68 \pm 13$ & $65 \pm 11$ & $65 \pm 10$ \\
\hline Mean number of VPCs per hour (median) & $20 \pm 38(4)$ & $28 \pm 40(12)$ & $24 \pm 35(7)$ & $15 \pm 40(3)$ \\
\hline Mean turbulence onset $(n=139), \%$ & $-0.0053 \pm 0.0264$ & $-0.0 \pm 0.024 I$ & $-0.006 \pm 0.0286$ & $-0.0129 \pm 0.0212$ \\
\hline $\begin{array}{l}\text { Mean turbulence slope }(n=139) \text {, } \\
\text { msec per RR interval }\end{array}$ & $6.411 \pm 4.684$ & $3.4716 \pm 3.640$ & $5.673 \pm 5.738$ & $7.354 \pm 5.984$ \\
\hline
\end{tabular}

Note: Percentages refer to $\%$ of total and are rounded to nearest half percent.

Abbreviations: ACS, acute coronary syndrome; VPC, ventricular premature contraction; NSTEMI, non-ST elevation myocardial infarction.

predictors of one-year outcome in patients for whom the scores could be computed (turbulence onset, hazard ratio 2.100, 95\% CI 1.340-3.292, $P=0.001$; turbulence slope, hazard ratio $2.302,95 \% \mathrm{CI} 1.459-3.632, P<0.001 ; \mathrm{n}=139$, 79 events). Turbulence slope was a significant predictor of 30-day outcomes (hazard ratio $2.704,95 \%$ CI 1.214-6.020, $P=0.015)$, but turbulence onset was not.

Table 2 Cardiovascular outcomes

\begin{tabular}{ll}
\hline 30-day cardiovascular outcomes & \\
- Returned to ED & $21(13 \%)$ \\
& (I6 patients were admitted to hospital from ED) \\
- Died & $5(3 \%)$ \\
- Total 30-day cardiovascular outcomes & $26(16 \%)$ \\
One-year cardiovascular (including 30-day) outcomes & \\
- Returned to ED & $80(48 \%)$ \\
& $(60$ patients were admitted to hospital from the ED) \\
- Died & $9(5 \%)$ \\
- Total one-year cardiovascular outcomes & $89(54 \%)$ \\
\hline
\end{tabular}

Abbreviation: ED, emergency department.
To compute turbulence risk scores, the 27 patients without turbulence onset or turbulence slope measurements due to too few VPCs were placed in the low-risk group in accordance with standards. ${ }^{7}$ Results of the TIMI and heart rate turbulence risk scores in relation to 30-day and one-year outcomes examined in univariate Cox regression models are reported in Table 5. Multivariate models with both TIMI and heart

Table 3 Frequency of patients within each category of Thrombolysis In Myocardial Infarction (TIMI) risk score

\begin{tabular}{lll}
\hline TIMI category & $\begin{array}{l}\text { Patients in category } \\
(\mathbf{n})\end{array}$ & $\begin{array}{l}\text { Percentage of total } \\
(\mathbf{n}=\mathbf{1 6 6})\end{array}$ \\
\hline 0-I lowest risk & 9 & 5.4 \\
2 low risk & 17 & 10.2 \\
3 medium-low risk & 60 & 36.1 \\
4 medium risk & 49 & 29.5 \\
5 medium-high risk & 28 & 16.9 \\
6-7 high risk & 3 & 1.8 \\
Total & 166 & 100.0 \\
\hline
\end{tabular}


Table 4 Frequency of patients within each category of heart rate turbulence risk score

\begin{tabular}{llll}
\hline $\begin{array}{l}\text { HRT } \\
\text { category }\end{array}$ & Score parameters & $\begin{array}{l}\text { Patients } \\
\text { in category } \\
\text { (n) }\end{array}$ & $\begin{array}{l}\text { Percentage } \\
\text { of total } \\
(\mathbf{n}=166)\end{array}$ \\
\hline Low risk & $\begin{array}{l}\text { TO }<0 \% \text { and TS }>2.5 \\
\text { msec per RR interval or } \\
\text { too few VPCs to calculate }\end{array}$ & I0I & 60.8 \\
Medium & $\begin{array}{l}\text { TO }>0 \% \text { or TS }<2.5 \\
\text { msec per RR interval }\end{array}$ & 43 & 25.9 \\
risk & $\begin{array}{l}\text { TO }>0 \% \text { and TS }<2.5 \\
\text { High risk }\end{array}$ & 22 & 13.3 \\
Totals & msec per RR interval & 166 & 100.0 \\
\hline
\end{tabular}

Abbreviations: HRT, heart rate turbulence; TO, turbulence onset; TS, turbulence slope; VPC, ventricular premature contraction.

rate turbulence risk scores together in relation to 30-day and one-year outcomes are presented in Table 6.

The TIMI risk score categories were divided into three groups for visual comparison of the three categories of heart rate turbulence risk score. Because categories 0-3 were closely aligned, category 4 was medium risk, and 5-6 showed the highest risk in our sample, the divisions were created accordingly. A Cox regression model examining the oneyear outcomes is shown in Figure 1 (model statistics are: chi square $31.263, P<0.001$, C-statistic 0.687 ; heart rate turbulence risk score hazard ratio $1.870,95 \%$ CI $1.428-2.448$, $P<0.001$; TIMI risk score hazard ratio $1.535,95 \% \mathrm{CI}$ 1.176-2.003, $P=0.002$ ).

The heart rate turbulence risk score, again including all 166 patients, was then examined in a multivariate Cox regression model with the TIMI score parameters included as seven separate variables. In relation to 30-day outcomes, the overall model was significant (chi square 18.037, $P=0.021$ ), and the turbulence risk score was the most significant independent

Table 5 Comparison of heart rate turbulence and TIMI risk scores in univariate Cox regression $(n=166)$

\begin{tabular}{|c|c|c|c|c|}
\hline $\begin{array}{l}\text { Risk score } \\
\text { measure }\end{array}$ & $\begin{array}{l}\text { Model } \\
\text { Chi square }\end{array}$ & $\begin{array}{l}\text { Model } \\
\text { P value }\end{array}$ & $\begin{array}{l}\text { HR } \\
(95 \% \mathrm{Cl})\end{array}$ & C-statistic \\
\hline \multicolumn{5}{|c|}{ Outcomes at 30 days } \\
\hline HRT risk & 7.923 & 0.005 & $\begin{array}{l}1.911 \\
(1.198-3.048)\end{array}$ & 0.647 \\
\hline TIMI risk & 6.702 & 0.010 & $\begin{array}{l}1.613 \\
(1.121-2.320)\end{array}$ & 0.687 \\
\hline \multicolumn{5}{|c|}{ Outcomes at one year } \\
\hline HRT risk & 22.553 & $<0.001$ & $\begin{array}{l}1.851 \\
(1.42-2.41)\end{array}$ & 0.657 \\
\hline TIMI risk & 9.905 & 0.002 & $\begin{array}{l}1.371 \\
(1.13-1.67)\end{array}$ & 0.605 \\
\hline
\end{tabular}

Abbreviations: $\mathrm{Cl}$, confidence interval; $\mathrm{HRT}$, heart rate turbulence; $\mathrm{HR}$, hazards ratio; TIMI, Thrombolysis In Myocardial Infarction.
Table 6 Heart rate turbulence and TIMI risk scores in multivariate Cox regression $(n=166)$

\begin{tabular}{|c|c|c|c|}
\hline \multicolumn{4}{|c|}{$\begin{array}{l}\text { Single model with HRT and TIMI risk scores: adverse } \\
\text { outcomes at } 30 \text { days ( } 26 \text { events) }\end{array}$} \\
\hline \multicolumn{4}{|c|}{ Model chi square $($ model $P$ value $)=13.200(0.001)$} \\
\hline \multicolumn{4}{|c|}{ Model C-statistic $=0.725$} \\
\hline $\begin{array}{l}\text { Risk score } \\
\text { measure }\end{array}$ & $\begin{array}{l}\text { Wald } \\
\text { statistic }\end{array}$ & $\begin{array}{l}\text { Variable } \\
P \text { value }\end{array}$ & $\begin{array}{l}\text { HR } \\
(95 \% \mathrm{Cl})\end{array}$ \\
\hline HRT risk & 7.572 & 0.013 & $1.818(1.33-2.915)$ \\
\hline TIMI risk & 3.352 & 0.018 & $1.570(1.080-2.283)$ \\
\hline
\end{tabular}

Single model with HRT and TIMI risk scores: adverse outcomes at one year ( 89 events)

Model chi square $($ model $P$ value $)=31.089(<0.00 \mathrm{I})$

Model C-statistic $=0.695$

\begin{tabular}{llll}
$\begin{array}{l}\text { Risk score } \\
\text { measure }\end{array}$ & $\begin{array}{l}\text { Wald } \\
\text { statistic }\end{array}$ & $\begin{array}{l}\text { Variable } \\
\text { P value }\end{array}$ & $\begin{array}{l}\text { HR } \\
(\mathbf{9 5} \% \mathbf{C I})\end{array}$ \\
\hline HRT risk & 20.776 & $<0.00 \mathrm{I}$ & $\mathrm{I} .860(\mathrm{I} .424-2.428)$ \\
TIMI risk & 9.667 & 0.002 & $\mathrm{I} .383(\mathrm{I} .127-\mathrm{I} .696)$
\end{tabular}

Abbreviations: $\mathrm{Cl}$, confidence interval; $\mathrm{HRT}$, heart rate turbulence; HR, hazards ratio; TIMI, Thrombolysis In Myocardial Infarction.

predictor in the model (hazard ratio $1.843,95 \%$ CI $1.107-$ $3.069, P=0.019)$. The only other variable that remained a significant predictor was two severe episodes of angina within the previous 24 hours (hazard ratio $0.318,95 \% \mathrm{CI}$ $0.105-0.958, P=0.042$ ). In relation to one-year outcomes, the turbulence risk score was again the most significant predictor (hazard ratio 1.908, 95\% CI 1.433-2.542, $P<0.001$ ). More than one severe episode of angina within the previous 24 hours (hazard ratio $0.588,95 \%$ CI $0.350-0.986$, $P=0.046)$ and use of aspirin within the previous seven days (hazard ratio $0.57896 \% \mathrm{CI} 0.343-0.975, P=0.040$ ) were also significant independent predictors. Examining the TIMI parameters separately in a multivariate model improved the C-statistic marginally to 0.735 in relation to 30 -day outcomes and 0.724 in relation to one-year outcomes.

\section{Discussion}

Our findings offer evidence that heart rate turbulence measured early in the emergency department has an accuracy approximately equivalent to the TIMI risk score in predicting 30-day and one-year outcomes. Since Schmidt et al showed that absence of heart rate turbulence was a significant predictor of total mortality in 100 patients with coronary heart disease, ${ }^{6}$ numerous studies have expanded those findings to aid in physiological understanding ${ }^{15-18}$ and clinical application..$^{8-10,19-22}$ However, optimal timing for clinical assessment of heart rate turbulence has not been established. ${ }^{7}$ In the study by Schmidt et al, heart rate turbulence was measured during a stable period from two weeks 

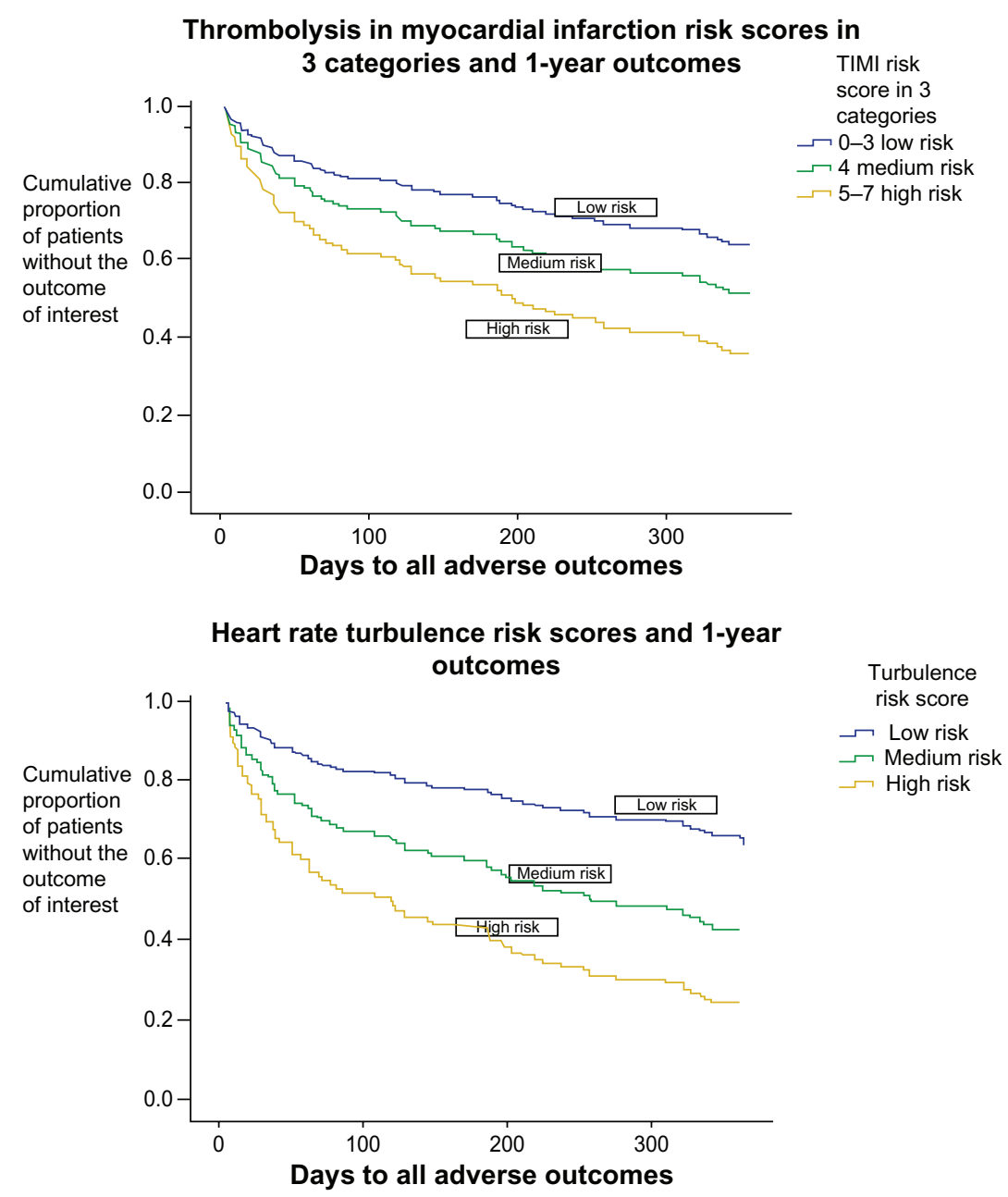

Figure I Comparison of TIMI and heart rate turbulence risk scores in acute coronary syndrome. Outcomes of emergent cardiac readmission or cardiac death at one year. Notes: In total, $100 \%$ of patients (proportionately equal to I.0) start at time zero (time of emergency department discharge) without an adverse event, ie, event-free (upper left hand corner). The lines represent the proportion of patients in each group who are event-free at the end of one year.

Abbreviation: TIMI, Thrombolysis In Myocardial Infarction.

up to three months after patients experienced a myocardial infarction. ${ }^{6}$ To investigate the relationship between heart rate turbulence and heart rate and test this association in acute myocardial infarction survivors, Bauer et al examined heart rate turbulence in patients 2-3 weeks after myocardial infarction. ${ }^{8}$ Huikuri et $\mathrm{al}^{21}$ measured heart rate turbulence at two time periods after myocardial infarction in two patient groups, ie, early (defined after 5-21 days in the first cohort and 2-4 weeks in the second) and late (at six weeks in the first cohort and 10-14 weeks in the second). The investigators found that heart rate turbulence improved over time, and that both time periods for measurement were prognostic of ventricular arrhythmias and fatal or near-fatal arrhythmic events. Sade et al measured heart rate turbulence immediately after admission to the coronary care unit, within six hours of symptom onset, in patients with myocardial infarction. ${ }^{9}$ In the study of patients with unstable angina by Lanza et al, 24-hour Holter recordings were started within 24 hours of hospital admission. ${ }^{10}$ Both Sade et al and Lanza et al found heart rate turbulence to be highly prognostic of adverse outcomes, including death six months to one year after an episode of acute coronary syndrome. While optimal timing of assessment of heart rate turbulence was not an aim in our study, we did establish that heart rate turbulence measured from 24-hour recordings started early after arrival in the emergency department was associated with outcomes up to one year after the patient's visit.

A high proportion of patients in our study were readmitted to hospital for cardiac reasons (48\%) over the course of one year compared with the literature, in which a rate of $30 \%$ is cited. ${ }^{22}$ However, more than two in three patients had a previous diagnosis of coronary artery disease, and over one in four had a comorbidity of diabetes, reflecting an unwell group of people with advanced disease and/or comorbidities, 
possibly contributing to the relatively high percentage of adverse outcomes. In any event, readmittance to hospital after an emergency department visit at which acute coronary syndrome is diagnosed is common, and contributes directly to the economic burden of health care. Johnston et al reported that even one cardiac rehospitalization within a year of presentation to the emergency department with acute coronary syndrome increased direct care costs by nearly $\$ 10,000 .{ }^{12}$ To be sure, return to the emergency department and rehospitalization in itself is not a reflection of quality of care. A structured, decision-making approach has been found to be safe and cost-effective, and is recommended. ${ }^{1}$ An additional risk assessment tool, such as heart rate turbulence, could be a practical choice to help health care providers identify NSTEMI and patients with unstable angina with an increased risk of 30-day or one-year adverse cardiac events.

The C-statistic indicates the ability of a statistical model to predict an outcome, and can be used for multivariable models, providing information similar to the area under the curve for univariate models. A C-statistic value of 0.5 would indicate that the model has a $50 \%$ chance of predicting an accurate outcome, or in other words, provide no additional information. The C-statistic scores in our TIMI and heart rate turbulence risk score univariate models in relation to both outcomes range from $60.5 \%$ (TIMI risk score as a predictor of one-year outcome) to $68.7 \%$ (TIMI risk score as a predictor of 30-day outcome). The heart rate turbulence risk score provided an accurate estimate in $64.7 \%$ of patients for 30 -day outcomes and $65.7 \%$ for one-year outcomes. In a multivariate model, the TIMI and heart rate turbulence risk scores together increased predictive ability to $72.5 \%$ for 30 -day outcomes and $69.5 \%$ for one-year outcomes. Figure 1 provides a visual comparison of these data. While this increase is only incremental, the results do indicate that the predictive ability of the heart rate turbulence risk score is similar to that of the TIMI risk score. In cases for which TIMI parameters may be unknown or their acquisition delayed, the heart rate turbulence risk score may provide a meaningful alternative, particularly given that this parameter can be obtained within the first 24 hours by routine ECG monitoring. To our knowledge, this is the first study to examine heart rate turbulence calculated from ECG recordings starting within the first hour of presentation to the emergency department in patients who arrive with symptoms of acute coronary syndrome and are subsequently diagnosed with unstable angina or NSTEMI.

The TIMI score is used for assessing patients' prognosis and assisting in clinical decision-making. Predictive accuracy for death or new myocardial infarction at one year has been examined with the aim of identifying patients who may benefit from close monitoring or more aggressive therapy. ${ }^{1,23}$ The seven variables, including elements of the patient's medical history that are needed to establish a TIMI score, may not always be immediately available. Heart rate turbulence could possibly serve as a noninvasive alternative, because it is a measurement derived from routine ECG monitoring that does not require multiple parameters to compute. Heart rate turbulence may also serve as a reasonable adjunct and/or alternative means to aid risk stratification of patients with unstable angina or NSTEMI if measured early, from the time they enter the emergency department with cardiac symptoms.

From a pragmatic perspective, heart rate turbulence might be computed during hospitalization, given that continuous 24-hour ECG monitoring for patients with acute coronary syndrome is recommended, ${ }^{1}$ and cardiac patients are usually monitored from the moment they enter the emergency department. With many patients staying in the hospital for only 24 hours (25\% in our sample; the remaining $75 \%$ stayed longer), the importance of gaining as much prognostic information as possible in a limited amount of time cannot be overemphasized.

\section{Limitations}

This was a retrospective analysis of data collected from early 2002 through 2004. Practice standards for management of acute coronary syndrome have been updated in the intervening time, which could have changed patients' trajectories. The study sample was small and underpowered to examine mortality alone at a hard endpoint. Analysis was hampered by ECG artifact and insufficient ECG recording time, this resulted in many recordings being left out of the analysis, which could reflect potential challenges to accurate assessment. In addition, heart rate turbulence requires sinus beats to compute, so patients with atrial fibrillation could not be included. Some of the follow-up data was obtained by phone calls to patients or family members. While every effort was made to confirm the information that was provided, not all records were available to support the stated reason for rehospitalization and/or death. The TIMI risk score was originally developed to predict two-week outcomes, so relating it to 30-day and one-year outcomes may be less sensitive and specific than the timeframe for which the instrument was designed.

\section{Conclusion}

Our preliminary findings suggest that evaluation of heart rate turbulence to assist in identifying patients with unstable 
angina or NSTEMI at high risk for adverse cardiac events over the course of 30 days or one year may have potential as a practical addition to continuous ECG monitoring. Heart rate turbulence could possibly serve as an independent predictor of cardiovascular events in patients with acute coronary syndrome without ST elevation because the 30-day and one-year predictive ability seems to be similar to the TIMI risk score. Future research could aim to examine prospectively the value of early measurement of heart rate turbulence, starting at the time patients with symptoms of acute coronary syndrome enter the emergency department.

\section{Acknowledgments}

The authors are grateful to the team at the Drew Electrocardiographic Monitoring Research Laboratory, the University of California, San Francisco School of Nursing, and the investigators who performed the IMMEDIATE AIM study; the research team at the Heart Rate Variability Laboratory, School of Medicine, Washington University, St Louis, MO, USA; and the patients who agreed to participate in this research.

\section{Disclosure}

The authors have no relevant financial or nonfinancial relationships to disclose in this work.

\section{References}

1. Anderson JL, Adams CD, Antman EM, et al. 2011 ACCF/AHA focused update of the guidelines for the management of patients with unstable angina/non ST-elevation myocardial infarction. Circulation. 2011;123:e426-e579.

2. Antman EM, Cohen M, Bernink PJ, et al. The TIMI risk score for unstable angina/non-ST elevation MI: a method for prognostication and therapeutic decision making. JAMA. 2000;284:835-842.

3. Pollack CV Jr, Sites FD, Shofer FS, Sease KL, Hollander JE. Application of the TIMI risk score for unstable angina and non-ST elevation acute coronary syndrome to an unselected emergency department chest pain population. Acad Emerg Med. 2006;13:13-18.

4. Hess EP, Agarwal D, Chandra S, et al. Diagnostic accuracy of the TIMI risk score in patients with chest pain in the emergency department: a meta-analysis. CMAJ. 2010;182:1039-1044.

5. de Araújo Gonçalves P, Ferreira J, Aguiar C, Seabra-Gomes R. TIMI, PURSUIT, and GRACE risk scores: sustained prognostic value and interaction with revascularization in NSTE-ACS. Eur Heart J. 2005; $26: 865-872$
6. Schmidt G, Malik M, Barthel P, et al. Heart-rate turbulence after ventricular premature beats as a predictor of mortality after acute myocardial infarction. Lancet. 1999;353:1390-1396.

7. Bauer A, Malik M, Schmidt G, et al. Heart rate turbulence; standards for measurement, physiological interpretation, and clinical use. $J \mathrm{Am}$ Coll Cardiol. 2008;52:1353-1365.

8. Bauer A, Malik M, Barthel P, et al. Turbulence dynamics: an independent predictor of late mortality after acute myocardial infarction. Int $J$ Cardiol. 2006;107:42-47.

9. Sade E, Aytemir K, Oto A, et al. Assessment of heart rate turbulence in the acute phase of myocardial infarction for long-term prognosis. Pacing Clin Electrophysiol. 2003;26:544-550.

10. Lanza GA, Sgueglia FA, Angeloni G, et al. Prognostic value of heart rate turbulence and its relation to inflammation in patients with unstable angina. Am J Cardiol. 2009;103:1066-1072.

11. Drew BJ, Schindler DM, Zegre JK, Fleishmann KE, Lux RL. Estimated body surface potential maps in emergency department patients with unrecognized transient myocardial ischemia. $J$ Electrocardiol. 2007;40:S15.

12. Johnston SS, Curkendall S, Makenbaeva D, et al. The direct and indirect cost burden of acute coronary syndrome. J Occup Environ Med. 2011;53:2-7.

13. Steyerberg E, Harrell FE Jr. Statistical models for prognostication. In: Interactive Textbook for Clinical Symptom Research. Bethesda, MD: National Institutes of Health; 2003. Available from: http:// painconsortium.nih.gov/symptomresearch/chapter_8/index.htm. Accessed May 3, 2013.

14. Steyerberg, EW. Bootstrap resampling. In: Clinical Prediction Models: A Practical Approach to Development, Validation, and Updating. New York, NY: Springer; 2009.

15. Wichterle D, Melenovsky V, Malik M. Mechanisms involved in heart rate turbulence. Card Electrophysiol Rev. 2002;6:262-266.

16. Wichterle D, Melenovsky V, Simek J, Malik J, Malik M. Hemodynamics and autonomic control of heart rate turbulence. J Cardiovasc Electrophysiol. 2006;17:286-291.

17. Bauer A, Schmidt G. Last piece of the heart rate turbulence puzzle? Heart Rhythm. 2007;4:290-291.

18. Zuern CS, Barthel P, Bauer A. Heart rate turbulence as a risk predictor after myocardial infarction. Front Physiol. 2011;2:99.

19. Ghuran A, Reid F, La Rovere MT, Schmidt G, Bigger JT, Camm J. Heart rate turbulence-based predictors of fatal and nonfatal cardiac arrest (The Autonomic Tone and Reflexes after Myocardial Infarction Substudy). Am J Cardiol. 2002;89:184-190.

20. Stein PK, Deedwania P. Usefulness of abnormal heart rate turbulence to predict cardiovascular mortality in high-risk patients with acute myocardial infarction and left ventricular dysfunction (from the EPHESUS study). Am J Cardiol. 2009;103:1495-1499.

21. Huikuri HV, Exner DV, Kavanagh KM, et al. Attenuated recovery of heart rate turbulence early after myocardial infarction identifies patients at high risk for fatal or near-fatal events. Heart Rhythm. 2010;7:229-235.

22. Miwa Y, Miyakoshi M, Hoshida K, et al. Heart rate turbulence can predict cardiac mortality following myocardial infarction in patients with diabetes mellitus. J Cardiovasc Electrophysiol. 2011;22:1135-1140.

23. Giugliano RP, Braunwald E. The year in non-ST-segment elevation acute coronary syndromes. J Am Coll Cardiol. 2005;46:906-919.
Vascular Health and Risk Management

\section{Publish your work in this journal}

Vascular Health and Risk Management is an international, peerreviewed journal of therapeutics and risk management, focusing on concise rapid reporting of clinical studies on the processes involved in the maintenance of vascular health; the monitoring, prevention and treatment of vascular disease and its sequelae; and the involvement of

\section{Dovepress}

metabolic disorders, particularly diabetes. This journal is indexed on PubMed Central and MedLine. The manuscript management system is completely online and includes a very quick and fair peer-review system, which is all easy to use. Visit http://www.dovepress.com/ testimonials.php to read real quotes from published authors. 